\title{
Study on the strain-compensated Arrhenius-type constitutive model of Mg-4AI-3Ca-1.5Zn-1Nd-0.2Mn Magnesium alloy
}

\author{
Gang Chen, An zhen Guo, Li Ma, Wei Chen, Guowei Zhang \\ Ningbo Branch of Ordnance Science Institute of China, Ningbo, 315103, China
}

Keywords: magnesium alloy, Arrhenius-type constitutive, strain-compensated.

\begin{abstract}
Compression tests of Mg-4Al-3Ca1.5Zn-1Nd-0.2Mn Magnesium alloy as-extruded were carried out on the Gleeble-3500 thermo-mechanical simulator. The compression testing temperatures were in the range of $200-350^{\circ} \mathrm{C}$ at intervals $50^{\circ} \mathrm{C}$, while the imposed constant true strain rates were $0.001,0.01,0.1,1$ and $10 \mathrm{~S}-1$.True stress-strain data were employed for constitutive analysis following the sine-hyperbolic Arrhenius equation. The related material constants $n, \alpha$ and $\beta$, as well as the activation energy $Q$ for each temperature regime have been determined considering the strain compensation using the cubic polynomial fitting method. And then, incorporating the strain compensation on the constitutive equations for experimental alloy is established.
\end{abstract}

\section{Introduction}

Due to the advantages of low density and acceptable strength properties, magnesium alloys have primary potential for lightweight structural application in automotive and aerospace industries [1-3]. However, it exhibits poor workability at room temperature because of hexagonal close-packed (HCP) crystal structure [4]. The constitutive equations may properly describe the flow behaviour of the materials. In order to predict the behaviour of $\mathrm{Mg}$ alloys during hot deformation, accurate constitutive equations are needed which describe the deformation characteristics of the alloy. A thorough study on hot deformation behaviour of the material is essential to properly design the hot deformation parameters, which directly affect the mechanical properties of the formed component and the microstructure evolution [5]. Hence, there are many studies on low behaviours of the Mg$\mathrm{Al}-\mathrm{Zn}$ series magnesium alloys manufactured by conventional process. However, very limited efforts have been paid to understand hot deformation behaviour of Mg-4Al-3Ca1.5Zn-1Nd-0.2Mn alloy manufactured by spray forming.

The object of this study is to investigate the general nature of the influence of strain, strain rate and forming temperature on the compressive deformation characteristics of experimental alloy. A comprehensive model describing the relationship of the flow stress, strain rate and temperature of the alloys at different temperatures is proposed by compensation of strain. The validity of descriptive results based on the proposed constitutive equation is also investigated.

\section{Experimental}

The experimental alloy billets as-deposited were fabricated by spray forming process on the OS10 spray forming equipment manufactured by Osprey company UK. The major elements excluding Mg contents (wt \%) are $4 \% \mathrm{Al}, 3 \% \mathrm{Ca}, 1.5 \% \mathrm{Zn}, 1 \% \mathrm{Nd}$ and $0.2 \% \mathrm{Mn}$, respectively. Cylinderical specimens for compression testing were machined to be height of $12 \mathrm{~mm}$ and a diameter of $10 \mathrm{~mm}$ from the as-received extruded rod in the extrusion direction. The hot compression tests were carried out on the Gleeble -3500 thermo-mechanical simulator. Prior to hot compression, the specimens were soaked at deformation temperature for $10 \mathrm{~min}$ to ensure a homogenous temperature distribution. The experimental temperatures were $200-350^{\circ} \mathrm{C}$ at interval $50^{\circ} \mathrm{C}$. At each deformation temperature, the constant strain rates of $0.001,0.01,0.1,1$ and10 s-1 were employed and the height of $12 \mathrm{~mm}$ specimens were isothermally compressed to be $6 \mathrm{~mm}$. After compression the specimens were rapidly quenched in water at room temperature. 


\section{Constitutive model and Constants solution}

\subsection{Constitutive model}

Constitutive equations are usually used to estimate the flow stresses of a material. The stressstrain data obtained from compression tests under different strain rates and temperatures. Those conditions can be used to determine the material constant of these equations. The correlation between the flow stress $\sigma$, temperature $\mathrm{T}$ and strain rate ${ }^{\dot{\varepsilon}}$, especially at high temperatures, could be widely expressed by Arrhenius type equation which is expressed as Eq. (1)-(4) [6-8].

$$
\begin{gathered}
\dot{\varepsilon}=A f(\sigma) \exp (-Q / R T) \\
\dot{\varepsilon}=A_{1} \sigma^{n_{1}} \exp (-Q / R T) \quad \text { for } a \quad \sigma<0.8 \\
\dot{\varepsilon}=A_{2} \exp (\beta \sigma) \exp (-Q / R T) \text { for } a \quad \sigma>1.2 \\
\dot{\varepsilon}=A_{3}[\sinh (\alpha \sigma)]^{n} \exp (-Q / R T) \text { for all } \sigma
\end{gathered}
$$

where $R$ is universal gas constant, $Q$ is the activation energy. $A 1, A 2, A 3, n, n 1, \alpha$ and $\beta$ are experimentally determined constants, $\alpha=\beta / n 1$, and $\sigma$ is the flow stress.

Furthermore, considering the effects of temperature and strain rate on material deformation behavior could be expressed by Zener-Holloman parameter (Z) in an exponent-type equation which is expressed as Eqs.(5)-(7)[9].

$$
\begin{gathered}
Z=\dot{\varepsilon} \exp (Q / R T) \\
Z=A_{3}[\sinh (\alpha \sigma)]^{n} \\
A_{3}[\sinh (\alpha \sigma)]^{n}=\dot{\varepsilon} \exp (Q / R T)
\end{gathered}
$$

\subsection{Constants solution}

Taking logarithm of both sides of Eq. (2) and Eq. (3) yields linear relationships as :

$$
\ln \dot{\varepsilon}=\ln \left(A_{1}\right)+n_{1} \ln \sigma-Q / R T
$$

$$
\ln \dot{\varepsilon}=\ln \left(A_{2}\right)+\beta \sigma-Q / R T
$$

The experimental data should yield linear lines at different temperatures. The slopes of the plots of Eq.(8) and Eq.(9) are the value of $n 1$ and $\beta$, respectively. According to $\alpha=\beta / n 1, \alpha$ value can be calculated. The same method can be taken for Eq.(7), and the linear formula can be expressed as Eq.(10). Bring $(\alpha \sigma)$ into Eq.(10), the $1 / \mathrm{n}$ and (Q/RT) values can be calculated by linear fitting at different strain rates and temperatures, respectively. According to the average intercept value (l(nA3- Q/TR)/n), we can calculate $\ln A 3$ value.

$$
\ln [\sinh (\alpha \sigma)]=\frac{1}{\mathrm{n}} \ln \dot{\varepsilon}+\frac{Q}{n R T}-\frac{\ln \left(A_{3}\right)}{\mathrm{n}}
$$

Similar procedure was repeated to find out values of materials constants at various strains in the range of 0.1-0.5 at intervals 0.1 (Tab. 1 ).

Tab 1: values of materials constants at various strains

\begin{tabular}{c|r|r|r|c|c}
\hline true & \multicolumn{5}{|c}{ material constants } \\
\cline { 2 - 6 } strain & $\beta$ & $\alpha$ & $\mathrm{n}$ & $\mathrm{Q}$ & $\ln \mathrm{A}_{3}$ \\
\hline 0.1 & 0.0561375 & 0.021526 & 1.746624 & 121.648 & 32.3554 \\
0.2 & 0.0629475 & 0.022955 & 1.838631 & 121.429 & 32.3992 \\
0.3 & 0.0678575 & 0.024149 & 1.917973 & 120.741 & 32.2262 \\
0.4 & 0.0708175 & 0.024939 & 1.975445 & 120.338 & 32.1066 \\
0.5 & 0.072195 & 0.026145 & 1.94401 & 121.415 & 32.4429 \\
\hline
\end{tabular}

\subsection{Strain compensation}

It is generally assumed that influence of strain on the constants in the formulas is insignificant 
and not considered in the formulas as mentioned above. However, it has been observed that $\alpha, \beta$, $\ln \mathrm{A} 3, \mathrm{n}$ and $\mathrm{Q}$ show insignificant variation with true strain in Fig. 1. Hence compensation of strain should be taken in to account to modify constitutive model that may accurately predict the flow stress. The influence of strain in the constitutive model is incorporated by assuming that the material constants are polynomial functions of strain [10-12].
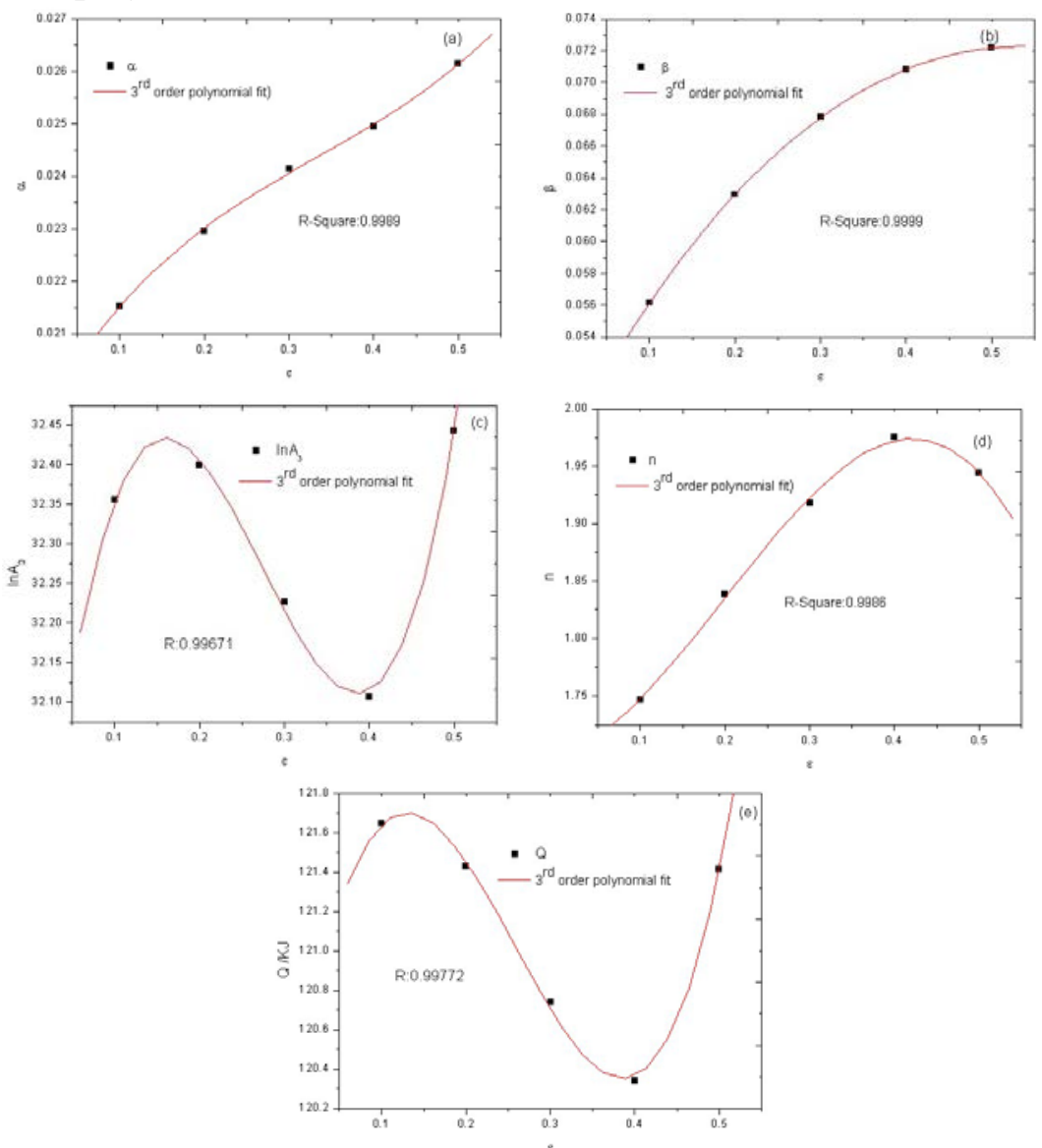

Fig. 1: Variation of (a) $\alpha$, (b) $\beta$, (c) $\ln \mathrm{A}_{3}$, (d) $n$ and (e) $Q$ with true strain (0.1-0.5)

The 3rd order polynomial fitting method was used to fit the relationship between different constants and true strain. The fitting curves show three variation tendencies (Fig. 1). the $\alpha$ value increase with increasing strain rapidly. The $\beta$ and $n$ values increase firstly and then decrease with increasing strain. The $\ln \mathrm{A} 3$ and $\mathrm{Q}$ values show " $\mathrm{N}$ " shape changing tendencies with increasing strain.

The fitted 3rd order polynomials were given by Eq. (11). The correlation coefficient (R) of the fitting curves were $0.9989,0.9999,0.9671,0.9986$ and 0.99772 , respectively. The results shown that 3rd order polynomials can represent the influence of material constants with good correlation and generalization (Fig. 1(a)-(e)).

$$
\left\{\begin{array}{l}
\alpha(\varepsilon)=0.01924+0.02767 \varepsilon-0.0549 \varepsilon^{2}+0.05426 \varepsilon^{3} \\
\beta(\varepsilon)=0.04714+0.10115 \varepsilon-0.11535 \varepsilon^{2}+0.02646 \varepsilon^{3} \\
\ln \mathrm{A}_{3}(\varepsilon)=31.71876+10.3761 \varepsilon-45.8925 \varepsilon^{2}+56.058333 \varepsilon^{3} \\
\mathrm{n}(\varepsilon)=1.69742+0.1839 \varepsilon+3.79848 \varepsilon^{2}-6.3535 \varepsilon^{3} \\
\mathrm{Q}(\varepsilon)=120.2912+24.4433 \varepsilon-125.625 \varepsilon^{2}+162.4166 \varepsilon^{3}
\end{array}\right.
$$

Once the material constants are evaluated, the strain-compensated Arrhenius-type constitutive model can express as following: 


$$
\left\{\begin{array}{l}
\dot{\varepsilon}=A_{3}(\varepsilon)[\sinh (\alpha(\varepsilon) \sigma)]^{n(\varepsilon)} \exp (-Q(\varepsilon) / R T) \\
\alpha(\varepsilon)=0.01924+0.02767 \varepsilon-0.0549 \varepsilon^{2}+0.05426 \varepsilon^{3} \\
\beta(\varepsilon)=0.04714+0.10115 \varepsilon-0.11535 \varepsilon^{2}+0.02646 \varepsilon^{3} \\
\ln A_{3}(\varepsilon)=31.71876+10.3761 \varepsilon-45.8925 \varepsilon^{2}+56.058333 \varepsilon^{3} \\
\mathrm{n}(\varepsilon)=1.69742+0.1839 \varepsilon+3.79848 \varepsilon^{2}-6.3535 \varepsilon^{3} \\
\mathrm{Q}(\varepsilon)=120.2912+24.4433 \varepsilon-125.625 \varepsilon^{2}+162.4166 \varepsilon^{3}
\end{array}\right.
$$

\section{Conclusions}

The flow stress data obtained from the hot compression tests over a wide range of temperatures (200-350 $\left.{ }^{\circ} \mathrm{C}\right)$, strain rates (0.001-1S-1) and strains (0.1-0.5) were used to study the strain compensation on the material constants to modify Arrhenius-type constitutive model. Strain has shown significant influence on materials constants. the $\alpha$ value increase with increasing strain rapidly. The $\beta$ and $n$ values increase firstly and then decrease with increasing strain. The $\ln A 3$ and $\mathrm{Q}$ values show " $\mathrm{N}$ " shape increasing tendencies with increasing strain. The strain-compensated for the materials constants of the constitutive mode was analyzed. Cubic polynomials were found to present the influence of strain on material constants with good correlation and generalization in terms of the adjusted coefficients of determination. The constitutive model is proposed based on the Arrhenius-type constitutive model considering the strain compensated.

\section{References}

[1] E. Cerri, P. Leo, P.P. De Marco. Hot compression behavior of the AZ91 magnesium alloy produced by high pressure die casting. Journal of Materials Processing Technology ,189,pp. 97-106, 2007.

[2] Yuan-Zhi Wu, Hong-Ge Yan, Ji-Hua Chen, et al. Hot deformation behavior and microstructure evolution of ZK21magnesium alloy. Materials Science and Engineering A, 527, pp. 36703675, 2010.

[3] K.P. Rao, Y.V.R.K. Prasad, K. Suresh, et al. Hot deformation behavior of Mg-2Sn-2Ca alloy in as-cast condition and after homogenization. Materials Science and Engineering A, 552, pp. 444- 450, 2012.

[4] M. Sivapragasha, P.R. Lakshminarayanan, R. Karthikeyan, et al. Hot deformation behavior of ZE41A magnesium alloy. Materials and Design, 29, pp. 860-866, 2008.

[5] O. Sivakesavam, Y.V.R.K. Prasad. Hot deformation behaviour of as-cast Mg-2Zn-1Mn alloy in compression: a study with processing map. Materials Science and Engineering A, 362, pp. 118-124, 2003.

[6] Songyi Chen, Kanghua Chen, Guosheng Peng, et al. Effect of heat treatment on hot deformation behavior and microstructure evolution of 7085 aluminum alloy. Journal of Alloys and Compounds, 537, pp. 338-345, 2012.

[7] M. Rajamuthamilselvan, S. Ramanathan. Hot deformation behaviour of 7075 alloy. Journal of Alloys and Compounds, 509, pp. 948-952, 2011.

[8] Nengping Jin, Hui Zhang, Yi Han, et al. Hot deformation behavior of 7150 aluminum alloy during compression at elevated temperature. Materials Characterization, 60, 530-536, 2009.

[9] Lei Xu, Guangze Dai, Xingmin Huang, et al. Foundation and application of Al-Zn-Mg-Cu alloy flow stress constitutive equation in friction screw press die forging. Materials and Design, 47, pp. 465-472, 2013.

[10] M.R. Rokni, A. Zarei-Hanzaki, Ali A. Roostaei, et al. Constitutive base analysis of a 7075 aluminum alloy during hot compression testing. Materials and Design, 32, pp. 4955-4960, 2011. 
[11] Y.C. Lin, L.-T. Li, Y.-Q. Jiang. A Phenomenological Constitutive Model for Describing Thermo-Viscoplastic Behavior of Al-Zn-Mg-Cu Alloy Under Hot Working Condition. Experimental Mechanics, 52, pp. 993-1002, 2012.

[12] Dipti Samantaray, C. Phaniraj, Sumantra Mandal, A.K. Bhaduri. Strain dependent rate equation to predict elevated temperature flow behavior of modified 9Cr-1Mo (P91) steel. Materials Science and Engineering A, 528, pp. 1071-1077, 2011. 